\title{
Situación laboral de los diplomados en biblioteconomía y documentación por la Universidad de Zaragoza (1992-2006)
}

\author{
Por María Domingo-del-Valle y María-Isabel Ubieto-Artur
}

\begin{abstract}
Resumen: Se analizan los resultados obtenidos a partir de 183 respuestas a una encuesta realizada a 660 diplomados en Biblioteconomía y Documentación de la Universidad de Zaragoza entre los años 1992 y 2006. Los resultados se pueden resumir en que estos profesionales incrementan sus resultados académicos con respecto a otros estudios anteriores debido a su motivación a lo largo de la carrera. Sus expectativas de inserción laboral se han cumplido en la mayoría de los casos, y su tiempo de acceso al mismo ronda los 13 meses de media. Una gran parte desempeña sus labores en funciones específicas de biblioteconomía y documentación. Sin embargo la mayor parte de ellos no pertenece a ninguna asociación profesional de ciencias de la documentación. Se concluye que estos estudios deberían promocionarse con el fin de que se identifique a estos profesionales con las tareas que son capaces de desempeñar.
\end{abstract}

Palabras clave: Profesionales de la información, Situación laboral, Oferta de empleo, Actividad profesional, Diplomados en biblioteconomía y documentación, Universidad de Zaragoza, Expectativas profesionales, Experiencia profesional.

Title: Employment survey of Universidad de Zaragoza graduates in Information Science studies

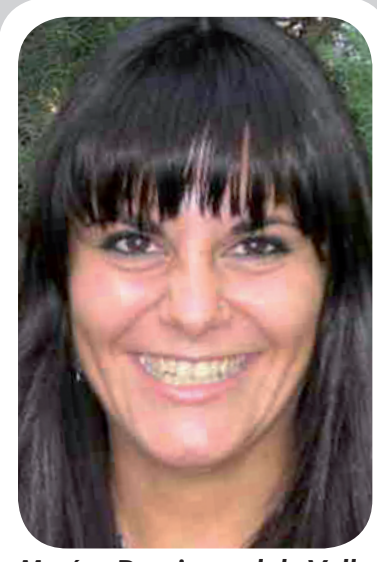

María Domingo-del Valle es diplomada en biblioteconomía y documentación por la Universidad de Zaragoza y es licenciada en documentación por la Universitat Oberta de Catalunya. Actualmente trabaja como documentalista en la Corporación Aragonesa de Radio y Televisión.

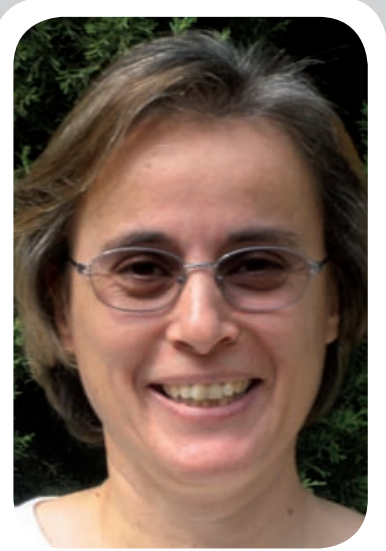

María-Isabel Ubieto-Artur es profesora titular de documentación general de la Universidad de Zaragoza y doctora en historia medieval. $\mathrm{Ha}$ trabajado en bibliotecas especializadas desde la década de los 80 e imparte diversas asignaturas de documentación en estudios de diplomatura, grado, doctorado y master.

Abstract: The article presents the results of a survey on working life, answered by 183 graduates in Information Science studies of the Universidad de Zaragoza, between the years 1992 and 2006 . The results can be summarized in that these professionals improve their academic results with respect to other previous studies due to their motivation throughout the studies. The expectations for job access of these graduates have been fulfilled in most of the cases, and they spent an average of thirteen months looking for a job. A great part of these workers are employed in specific functions of Information Science. Nevertheless, most of them don't belong to any Professional Association of Information Sciences. In spite of this, we can conclude that these studies must be better known in order to show everybody what opportunities are available to these professionals.

Keywords: Information Professionals, Labour market, Job access, Job vacancy, School-working life relations, Librarian and information professionals graduates, Universidad de Zaragoza (Spain), Working life; Professional experience.

Domingo-del-Valle, María; Ubieto-Artur, María-Isabel. "Situación laboral de los diplomados en biblioteconomía y documentación por la Universidad de Zaragoza (1992-2006)”. En: El profesional de la información, 2008, noviembrediciembre, v. 17, n. 6, pp. 648-655.

DOI: $10.3145 /$ epi.2008.nov.07

\section{Introducción}

Aunque "no existen estudios globales concluyentes sobre la inserción laboral de los titulados y la adecuación de su formación a las exigencias del mercado de trabajo", sí que hay algunos trabajos de universidades y asociaciones profesionales que permiten conocer dónde trabajan los titulados universitarios españoles. De la Uni- versidad de Zaragoza (Unizar), y concretamente de sus diplomados en biblioteconomía y documentación, sólo se ha publicado el artículo de Muñoz Escolá ${ }^{2}$ de 1997, lo que pone de relieve la necesidad de conocer cuál es la situación ahora. Conocerlo ayuda a diseñar con mayor congruencia el futuro título de grado en información y documentación de la Unizar. Con ese objetivo hemos realizado este trabajo, que surge de un proyecto de in- 
novación docente durante el curso 2006-2007, cuando se iniciaron otros estudios complementarios ${ }^{3}$.

El estudio recoge datos desde junio de 1992 cuando los primeros egresados accedieron al mercado de trabajo, si bien la diplomatura en biblioteconomía y documentación se oferta en la Facultad de Filosofía y Letras de la Unizar desde el curso académico 1989-1990.

\section{Metodología}

El trabajo parte de una encuesta anónima enviada por correo postal al domicilio familiar de los 660 diplomados que alcanzaron dicho grado entre junio de 1992 y septiembre de 2006 en la Unizar ${ }^{4}$.

Para su elaboración se tomó como modelo la encuesta enviada por el Decanato de la Facultad de Filosofía y Letras en 2005 a los alumnos de las 9 titulaciones de esta facultad ${ }^{5}$. En ella fueron encuestados 238 diplomados en biblioteconomía y documentación de los que sólo contestaron 45, un 18,91\%. Al incluir todas las titulaciones de la facultad, da una visión tan general que deja desdibujada la especificidad de nuestros titulados.

Nuestra encuesta se estructura en 75 preguntas agrupadas en dos apartados: la formación recibida antes y durante la carrera, y la inserción laboral y el desarrollo profesional.

La mayor parte de las cuestiones eran cerradas. En algunos casos se añadió la posibilidad de realizar observaciones, lo que ha provocado que a veces el nivel de precisión de las respuestas no permita comparaciones ${ }^{6}$. El envío de la encuesta se realizó el 19 de marzo de 2007 y las respuestas se recibieron entre el 23 de marzo y el 29 de junio. Las encuestas respondidas son 183 $(27,72 \%)$.

Las respuestas se introdujeron en una hoja de cálculo Excel y posteriormente en una base de datos en File Maker.

\subsection{Descripción del cuestionario}

Las preguntas se dividieron en subapartados para facilitar respuestas precisas:

a. Datos personales y académicos previos.

- Datos personales.

- Historial académico previo a la diplomatura.

b. Análisis del período educativo universitario.

- Estudios universitarios elegidos.

- Otras actividades formativas.

c. Actividades laborales.

- Empleo actual/último: Situación actual.
- Funciones y retribución del empleo actual/último.

d. Adecuación trabajo-estudios.

e. Observaciones.

Este artículo se limita a presentar los resultados del apartado c, correspondiente a las actividades laborales.

\section{Resultados}

La Unizar no cuenta con datos validados sobre el número de personas que se matricularon por primera vez en la diplomatura y el de aquéllas que obtuvieron el grado de diplomado durante las cuatro primeras promociones (entre 1989-1992 y 1992-1995). El resto de los datos, a partir de los que hemos obtenido los resultados que presentamos, se muestran en la tabla 1.

\subsection{Perfil general del diplomado}

Se trata de una mujer (78\%), que ha compaginado sus estudios con otras actividades entre las que destacan los trabajos remunerados. Proceden de la propia Comunidad de Aragón (71\%) o de otras, como Navarra (9\%), País Vasco (6\%), Castilla León ${ }^{7}(6 \%)$, La Rioja (5\%), Catalunya (1\%) y Otras (2\%) como la Comunidad Valenciana, Islas Baleares o Inglaterra.

\section{"El perfil del diplomado es mayoritariamente femenino $y$ ha compaginado estudios y trabajo"}

Los estudiantes aragoneses que cursaron la diplomatura procedían en el $88 \%$ de los casos de la provincia de Zaragoza ( $82 \%$ de la ciudad y $6 \%$ del resto de la provincia), $6 \%$ de la provincia de Huesca (4\% de la ciudad) y otro $6 \%$ de la de Teruel con un reparto equitativo entre la provincia y su capital.

Si comparamos el lugar de procedencia con la residencia actual de los diplomados comprobamos que la mayoría sigue residiendo en Aragón (63\%). El resto se distribuye de la siguiente manera: Navarra recupera sus profesionales una vez formados (9\%), a Madrid emigra el 8\%, a La Rioja vuelve el 5\%, el País Vasco pierde 1\% de sus habitantes (5\%), Catalunya mantiene el $1 \%$, y el $7 \%$ se desplaza a otros lugares de España ${ }^{8}$. Además el $2 \%$ de los diplomados emigran al extranjero a desarrollar su profesión?.

El acceso a la universidad ha sido mayoritariamente a través de la Logse o el COU con un $78 \%$ de los casos, frente a quienes accedieron tras cursar otras ca- 


\begin{tabular}{|c|c|c|c|c|}
\hline Promoción & $\begin{array}{l}\text { Matriculados } \\
\text { primer curso }\end{array}$ & $\begin{array}{l}\text { Diplomados } \\
\text { egresados }\end{array}$ & $\begin{array}{l}\text { Participantes } \\
\text { en el estudio }\end{array}$ & Porcentaje \\
\hline 1989-1992 & - & - & 0 & 0 \\
\hline 1990-1993 & - & - & 2 & 1 \\
\hline 1991-1994 & - & - & 2 & 1 \\
\hline 1992-1995 & - & - & 16 & 9 \\
\hline 1993-1996 & 145 & 91 & 18 & 10 \\
\hline 1994-1997 & 152 & 117 & 24 & 13 \\
\hline 1995-1998 & 142 & 96 & 22 & 12 \\
\hline 1996-1999 & 95 & 46 & 26 & 14 \\
\hline 1997-2000 & 107 & 92 & 16 & 9 \\
\hline 1998-2001 & 106 & 41 & 13 & 7 \\
\hline 1999-2002 & 109 & 23 & 18 & 10 \\
\hline 2000-2003 & 76 & 46 & 6 & 3 \\
\hline 2001-2004 & 67 & 53 & 5 & 3 \\
\hline 2002-2005 & 52 & 41 & 14 & 8 \\
\hline \multirow[t]{2}{*}{ 2003-2006 } & 51 & 14 & 1 & 1 \\
\hline & & 660 & 183 & 100 \\
\hline
\end{tabular}

--- Sin datos validados

Tabla 1. Diplomados participantes por promoción

rreras universitarias (diplomados o licenciados 9\%), FP o ciclos formativos (6\%), por ser mayores de 25 años (4\%), por traslado de otras carreras no finalizadas (2\%) o por otros medios $(1 \%)$.

La nota media de acceso a la carrera no ha sido respondida por el $10 \%$ de los participantes, porcentaje que aumenta hasta el $27 \%$ cuando se pregunta por la obtenida en la diplomatura. El 61\% restante ingresa en los estudios con una nota media de 6,5 o de 5,5 (en el $23 \%$ de los casos). El número de quienes ingresaron con una media de notable (13\% alcanzó el 7,5) o de sobresaliente es más reducido (el 2\% alcanzó una media de 9). El rendimiento académico de los diplomados sube a lo largo de su carrera, reduciéndose las calificaciones de aprobado y bien a favor de notable y sobresaliente. Si comparamos las notas medias de acceso y egreso comprobamos que la primera asciende desde el 6,3 al 6,8 en el caso de la nota media de los diplomados.

Los alumnos eligieron estos estudios en primera opción en el 77\% de los casos, un 16\% en segunda opción. La razón de la elección se distribuye entre una inclinación vocacional $(38 \%)$, por curiosidad $(23 \%)$, por recomendación (14\%), por no poder elegir la que deseaban $(10 \%)$, por su corta duración $(8 \%)$ o por otras razones $(7 \%)$, donde se encuentran opiniones muy variadas. Entre estas últimas podemos destacar: las salidas laborales (47\%), para obtener la titulación o poder optar a un segundo ciclo (20\%), porque no tenían nada claro $(20 \%)$ o porque era una titulación que se impartía en Zaragoza.

Cuando se pregunta si tras su experiencia formativa y profesional volverían a cursar la diplomatura, el 53\% afirma que sí, el $29 \%$ lo pone en duda y el $18 \%$ dice que no. Estas respuestas reflejan las afirmaciones anteriores donde más del 50\% había elegido la carrera por vocación o curiosidad, el $25 \%$ aproximadamente por recomendación o por no poder elegir la que deseaba y el resto se matriculó simplemente porque duraba sólo 3 años frente al resto de titulaciones o debido a las supuestas salidas laborales, que algunos de ellos no han visto cumplidas como deseaban ${ }^{10}$.

\subsection{Perfil de la actividad profesional}

Durante estos años los diplomados se han dedicado a un trabajo regular (45\%), varios temporales (27\%) o han ejercido como becarios (13\%). Sólo el 6\% se distribuye por igual entre quienes no han tenido trabajo o han ocupado este tiempo en otras actividades como preparar oposiciones.

A esta cuestión responde el $96 \%$ y afirman que se encuentran trabajando (93\%). Quienes trabajan se dividen entre el 55\% en el sector de la información y documentación y el $45 \%$ en otras áreas.

Los puestos relacionados con nuestro sector pue- 


\begin{tabular}{|c|c|c|}
\hline Especialidad & Denominación & № puestos \\
\hline \multirow[t]{6}{*}{ Archivo } & Auxiliar & 1 \\
\hline & Archivero & 1 \\
\hline & Becario & 1 \\
\hline & Gestión del archivo en una empresa & 1 \\
\hline & Técnico de archivo & 2 \\
\hline & & 6 \\
\hline \multirow[t]{8}{*}{ Bibliotecas } & Auxiliar & 13 \\
\hline & Ayudante & 4 \\
\hline & Becario & 1 \\
\hline & Bibliotecaria & 16 \\
\hline & Encargado de biblioteca & 2 \\
\hline & Facultativo Superior de Patrimonio Cultural (Bibliotecas) & 1 \\
\hline & Responsable de biblioteca & 2 \\
\hline & & 39 \\
\hline \multirow[t]{11}{*}{ Documentación } & Becario & 2 \\
\hline & Coordinador de departamento de documentación & 1 \\
\hline & Documentación especializada en el ámbito biomédico & 1 \\
\hline & Documentalista & 16 \\
\hline & Documentalista audiovisual & 2 \\
\hline & Documentalista y webmaster & 1 \\
\hline & Documentalista-catalogador & 4 \\
\hline & Jefe de negociado de documentación & 1 \\
\hline & Responsable de documentación & 1 \\
\hline & Técnico medio documentalista & 1 \\
\hline & & 30 \\
\hline Información y & Auxiliar técnico de bibliotecas y archivo & 1 \\
\hline \multirow[t]{9}{*}{ documentación } & Ayudante de archivo y biblioteca & 3 \\
\hline & Becario archivo, biblioteca y documentalista & 1 \\
\hline & Bibliotecaria / documentalista & 1 \\
\hline & Bibliotecario, documentalista, administrativo, archivo & 1 \\
\hline & Director de departamento de gestión de contenidos & 1 \\
\hline & Responsable de archivo y documentación & 1 \\
\hline & Técnico de documentación y bibliotecas & 1 \\
\hline & Gestor de la información & 1 \\
\hline & & 11 \\
\hline \multirow[t]{6}{*}{ Investigación } & Investigación archivística & 1 \\
\hline & Investigador en formación & 1 \\
\hline & Becario OTRI & 1 \\
\hline & Becario proyecto de investigación & 2 \\
\hline & Beca de colaboración & 1 \\
\hline & & 6 \\
\hline \multirow[t]{3}{*}{ Librero } & Librero & 1 \\
\hline & Dependienta de librería & 2 \\
\hline & & 3 \\
\hline Medios de & Locutor-redactor radio Sobrarbe, corresponsal de Heraldo & 1 \\
\hline \multirow[t]{2}{*}{ comunicación* } & Redactor / reportero gráfico & 1 \\
\hline & & 2 \\
\hline
\end{tabular}

* Desarrollan funciones de documentalistas

Tabla 2. Denominación de los puestos de trabajo

den desglosarse en distintas áreas que se muestran en la tabla $2^{11}$.

Los titulados que ejercen en otros sectores desempeñan actividades culturales y deportivas, venta al por mayor y comercio al por menor, educación superior, informática...

Sólo el $2 \%$ señala que no ha tenido ningún trabajo hasta la fecha. El 7\% de titulados que no tiene trabajo 


\begin{tabular}{|l|l|c|c|}
\hline \multicolumn{2}{|c|}{ Otros requerimientos } & No personas & \% personas \\
\hline \multirow{4}{*}{ I diomas } & Inglés & 49 & 12,7 \\
\cline { 2 - 4 } & Francés & 7 & 1,8 \\
\cline { 2 - 4 } & Alemán & 1 & 0,3 \\
\cline { 2 - 4 } & Otros idiomas & 11 & 2,8 \\
\hline \multirow{4}{*}{ Conocimientos informáticos } & Bases de datos & 71 & 18,4 \\
\cline { 2 - 4 } & Procesadores de texto & 71 & 18,4 \\
\cline { 2 - 4 } & Hojas de cálculo & 63 & 16,3 \\
\cline { 2 - 4 } & Sistemas operativos & 48 & 12,4 \\
\cline { 2 - 4 } & Lenguajes de programación & 12 & 3,1 \\
\cline { 2 - 4 } & Otros & 25 & 6,5 \\
\hline \multirow{2}{*}{ Otros requerimientos } & & 29 & 7,5 \\
\hline
\end{tabular}

Tabla 3. Requisitos exigidos a los diplomados

\section{"En el momento de realización de la encuesta la mayoría de los diplomados está trabajando (93\%)"}

y lo está buscando lleva una media de 9 meses en esta situación. Quienes trabajan dicen que tardaron una media de 13 meses en encontrar el último empleo.

La mayoría (44\%) de las 175 personas que han respondido sobre el número de trabajos han tenido 2 ó 3 de ellos a lo largo de su vida, y en el lado contrario, sólo un $15 \%$ ha desempeñado un único trabajo.
Como podemos comprobar en la figura 1, el año en el que más personas han iniciado su ocupación actual corresponde a 2006, con 47 personas. Le sigue 2003 con 23 titulados, y 2004 y 2005 con 18 diplomados.

La vía de acceso principal al último trabajo es por oposición o concurso público (31\%) seguida por los contactos personales o familiares (18\%), y por los anuncios en prensa (15\%) e internet (10\%). En menor medida aparece citado el Servicio de la Universidad o la Agencia Pública de empleo (6\%).

Los tipos de contrato mayoritarios son el laboral indefinido (35\%) y el laboral temporal (obra y servicio, interino, eventual) (33\%). Los funcionarios indefinidos suman el $15 \%$ y los becarios un $9 \%$. Otras formas

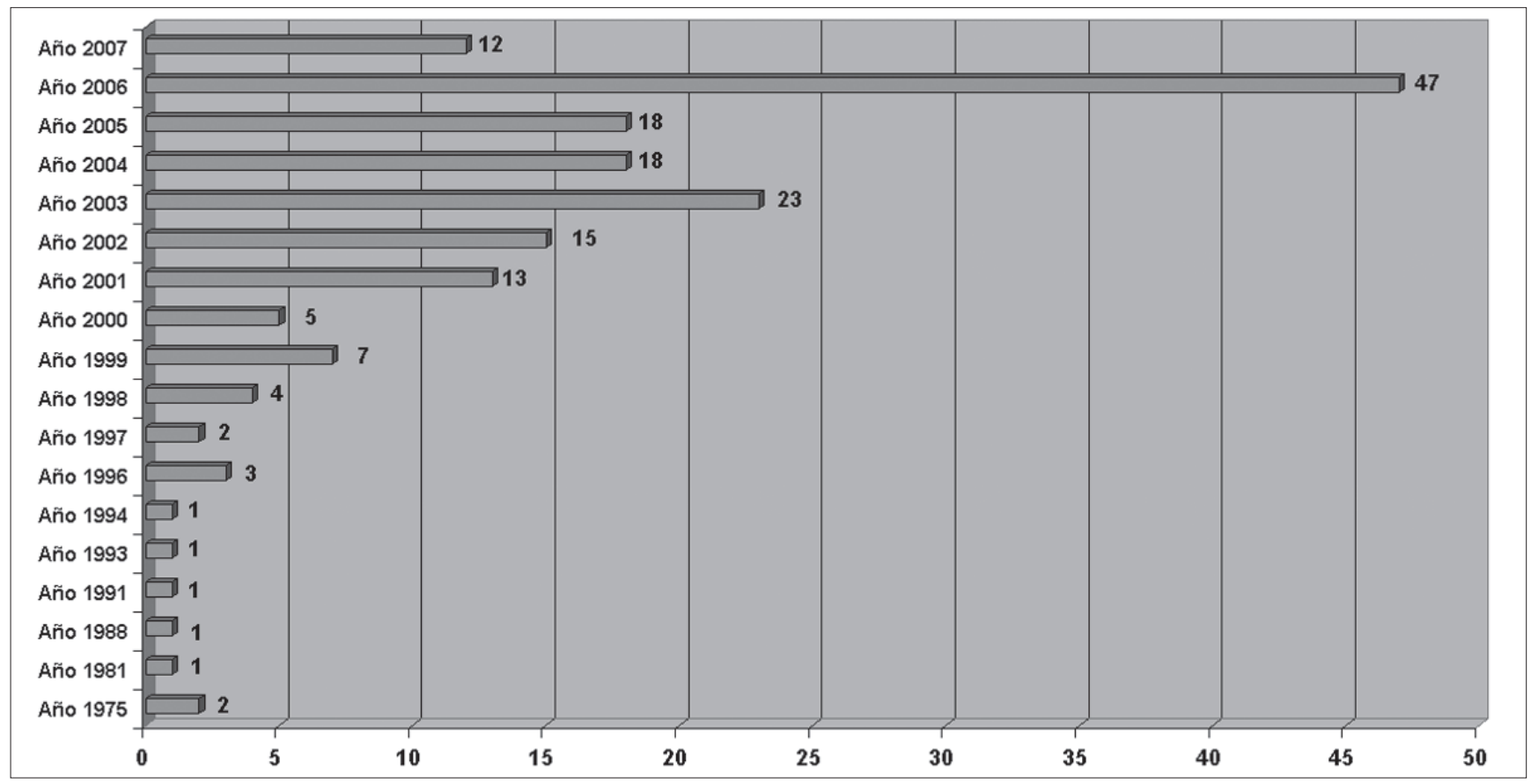

Figura 1. Año de inicio del trabajo actual (número de personas) 
registradas son el contrato administrativo, el contrato laboral temporal en prácticas y el empresario o trabajador por cuenta propia.

La jornada laboral habitual $(66 \%)$ es a tiempo completo, con dedicación de 35-40 horas semanales. En menor medida aparecen representadas la jornada de más de 40 horas semanales $(9 \%)$ y la de menos de 20 $(4 \%)$.

\subsection{Funciones y retribución}

El trabajo se reparte casi por igual entre el sector público $(52 \%)$ y el privado.

El lugar del puesto de trabajo es la comunidad autónoma de Aragón con un 63\%. Visto que al iniciar sus estudios el $71 \%$ procedía de la propia comunidad, significa que casi un $10 \%$ han "emigrado". Según muestran las cifras han ido a Navarra o Madrid, con un $8 \%$ respectivamente, o a las comunidades de La Rioja y País Vasco con un 5\% cada una.

Sus funciones abarcan desde la gestión documental, la catalogación, el mantenimiento de bases de datos y otras, como se observa en la figura 2.

La retribución bruta mensual mayoritaria (40\%) se encuentra entre los 900 y 1.300 euros. El 20\% cobra entre 500 y 900 , el $21 \%$ entre 1.400 y 1.800 , y sólo un $11 \%$ cobra más de 1.800 euros. Debemos aclarar aquí que el $4 \%$ del total trabaja menos de 20 horas semana$\operatorname{les}^{12}$.

La formación continua (la que se cursa con posterioridad a la obtención del título universitario) resulta imprescindible para alcanzar un puesto de trabajo acorde con la formación recibida o para promocionarse. Por ello sorprende comprobar que sólo el 58\% de los parti- cipantes afirma haber realizado cursos de especialización tras su graduación. Los cursos en los que se han matriculado estas personas se pueden agrupar en las áreas siguientes: tecnologías de la información (28\%), bibliotecas (26\%), documentación (21\%), archivos (14\%) y otros (11\%). Entre estos últimos se enumeran diversos cursos sobre economía, gestión financiera, procesos y procedimientos, gestión de pequeño comercio, comunicación de empresa, etc.

\subsection{Asociacionismo}

A pesar del importante papel que desarrollan las asociaciones profesionales en la actualización de conocimientos y el intercambio de experiencias, sólo el $21 \%$ de los diplomados afirma pertenecer a una. Anabad ha sido la elegida por el $56 \%$ de ellos, seguida de Sedic (13\%), la Asociación Vasca de Profesionales de Archivos, Bibliotecas y Centros de Documentación (Aldee) (10\%) y el Col-legi Oficial de Bibliotecaris-Documentalistes de Catalunya (Cobdc) ( $8 \%$ ). Una de las razones que puede influir en el bajo nivel de asociacionismo puede deberse a que las asociaciones de las distintas autonomías no ofertan lo que los profesionales creen necesitar y las de carácter nacional lo hacen preferentemente en Madrid. Esperamos que con la paulatina introducción de los cursos en línea y de asesorías jurídicas profesionales -puestas a disposición de los asociados- aumente el interés.

\section{"El $78 \%$ de los titulados no pertenece a ninguna asociación profesional de ciencias de la documentación"}

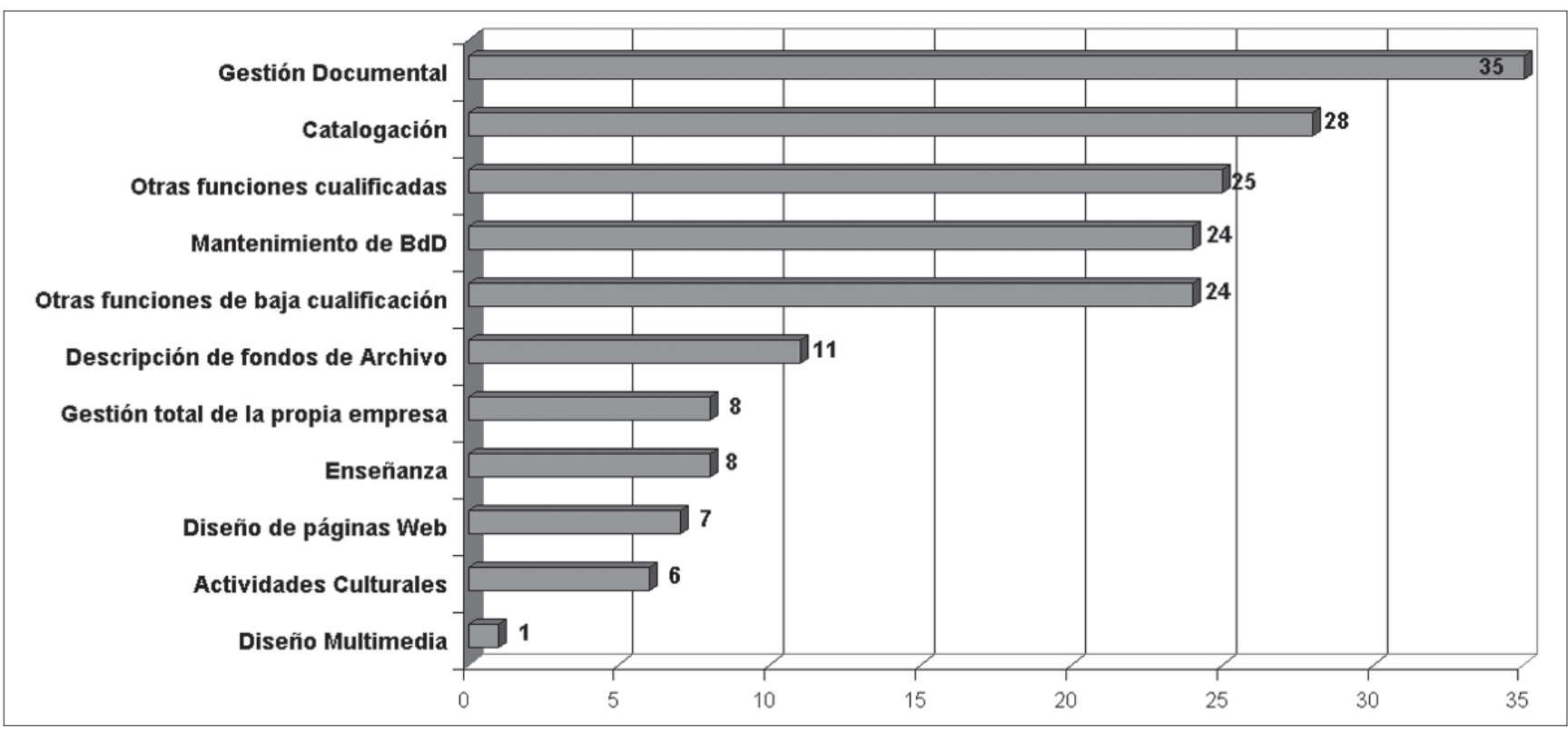

Figura 2. Funciones que realizan (número de casos) 


\subsection{Adecuación del trabajo a los estudios}

Según sus respuestas, la mayoría (52\%) ha podido acceder al puesto de trabajo por poseer una titulación universitaria de primer ciclo. Además el 35\% de estas plazas requerían específicamente la posesión del título de diplomado en biblioteconomía y documentación. En cambio en el $48 \%$ de los casos en que no era imprescindible contar con ninguna titulación, el 37\% considera que esta formación sí era necesaria para poder desarrollar correctamente las labores que se les encomendaron.

Al 52\% a quienes se ha exigido la titulación universitaria se les pedía además otros requisitos que incluye la tabla 3 .

Quienes están trabajando actualmente califican su trabajo así:

"Interesante" (20,4\%)

"Todavía no he encontrado un trabajo más apropiado" $(18,8 \%)$ $(17,7 \%)$

"No pertinente, no se corresponde a mis estudios"

\section{"Me proporciona seguridad" (16\%)}

"Me permite trabajar en la localidad que prefiero" $(14,4 \%), y$

"Me ofrece mejores perspectivas profesionales" $(12,7 \%)$

Problemas que encuentran al evaluar la adecuación de los contenidos de su carrera respecto a la inserción laboral:

"Es una carrera con escasa promoción universitaria"

"Carencia de especialización"

"Escasa formación complementaria" y

"Falta de espíritu empresarial".

Estos resultados se asemejan a los obtenidos en el estudio mencionado al inicio del artículo que incluía todas más titulaciones ${ }^{5}$. Además los participantes subrayan la ausencia de un colegio profesional como uno de los problemas para su formación continua y posterior inserción laboral. Como aspectos relevantes de la carrera, valorados en el mercado laboral, sobresalen la "formación general" y el "manejo de técnicas".

Sobre la adecuación de su situación laboral a las expectativas que tenían cuando empezaron sus estudios responden que se ajusta "totalmente" el $8 \%$, "mucho" el $12 \%$, "bastante" el $35 \%$ y negativamente el $45 \%$. Respuestas que están en sintonía con los resultados ob- tenidos sobre la necesidad o no de contar con una titulación universitaria y del mejor o peor puesto de trabajo conseguido.

\section{Conclusiones}

- La mayor parte de los diplomados ha elegido cursar esta carrera por motivos vocacionales o de cierta curiosidad, dado que en principio les atrae el mundo de la información, la lectura o internet. Por ello ha sido su primera opción a la hora de seleccionar su formación universitaria. Esta característica se ve reflejada en el aumento de la nota media obtenida tras el paso por la universidad.

- Existe un alto grado de colocación entre estos titulados, desempeñando la mayoría de ellos trabajos relacionados con su formación académica.

- Las áreas de trabajo cubiertas son las tradicionales (archivos, bibliotecas y centros y servicios de información especializados entre los que destacan los de los medios de comunicación), así como el apoyo a la investigación y el trabajo en librerías.

- La media de tiempo que tardan en encontrar trabajo ronda los trece meses.

- Los puestos de trabajo se distribuyen casi equitativamente entre sector público y privado, y las oposiciones o concursos públicos son las vías de acceso más utilizadas.

- Las denominaciones de los puestos de trabajo siguen siendo muy variadas, y no existe una normalización consensuada por parte de los empleadores. Esto puede dificultar la identificación de estos puestos por parte de los diplomados en biblioteconomía y documentación.

- La temporalidad en el trabajo parece ser una tónica generalizada. Esto da lugar a un alto número de trabajos desempeñados en un reducido número de años.

- Los tipos de contrato habituales son laboral indefinido y temporal (obra, servicio, interino o eventual).

- La jornada laboral que predomina es a tiempo completo y la retribución bruta mensual mayoritaria ronda los 900 a 1300 euros.

- En los últimos seis años se aprecia un aumento gradual de la inserción laboral de estos titulados.

- Existe una emigración de profesionales de ciertas comunidades autónomas, concretamente de Aragón y de Castilla y León, hacia la Comunidad de Madrid y otras como Navarra, País Vasco y Catalunya así como al extranjero. Esta fuga de profesionales se va reduciendo según se va extendiendo el desarrollo de la sociedad de la información y la dotación de plazas para ellos en las 
administraciones públicas y el sector privado en las comunidades autónomas de las que proceden. Nos encontramos por tanto con unos profesionales cuya inserción laboral depende directamente del grado de desarrollo de la sociedad de la información y el conocimiento en las distintas regiones.

\section{Notas}

1. Título de grado en información y documentación. Madrid: Agencia Nacional de Evaluación de la Calidad y Acreditación, 2004, p. 39. Consultado en: 12-09-07.

http://www.aneca.es/activin/docs/libroblanco_jun05_documentacion.pdf

2. Muñoz-Escolá, María-Mercedes. "Análisis de la situación laboral y de formación de los estudiantes y diplomados en biblioteconomía y documentación por la Universidad de Zaragoza”. En: Revista general de información y documentación, 1997, v. 7, n. 1, pp. 329-344.

3. Ubieto-Artur, María-Isabel; García-Marco, Francisco-Javier; GayMolins, Ma-del-Pilar; Ubieto-Artur, Antonio-Paulo; Velasco-de-laPeña, Esperanza. "Proyecto de innovación docente para la adaptación de la diplomatura en biblioteconomía y documentación (1 curso) al Espacio Europeo de Educación Superior". En: Innovación docente, tecnologías de la información y la comunicación e investigación educativa en la Universidad de Zaragoza. Caminando hacia Europa. Zaragoza: la Universidad, 2007. ISBN 978-84-96214-85-9. Consultado en: 12-09-07.

http://www.unizar.es/eees/innovacion06/COMUNIC_PUBLI/BLOQUE_I/ CAP_I_11.pdf

4. Queremos agradecer a la Facultad de Filosofía y Letras la colaboración en este estudio, ya que gracias a su ayuda pudimos disponer del listado de las direcciones de los 660 egresados durante este período, así como de los datos correspondientes al año de comienzo y finalización de la carrera, lo que nos ha permitido comparar su situación y recorrido en la titulación y en la facultad. Asimismo agradecemos a los 183 diplomados que han participado en este estudio. Sin su colaboración este trabajo no podría haberse realizado.

5. Análisis de la inserción laboral y expectativas profesionales de los graduados en Filosofía y Letras en la Universidad de Zaragoza (1999-2005). Zaragoza: la Facultad, 2005. Consultado en: 12-09-07.

http://fyl.unizar.es/doc/Publicacion_egresados_Def.pdf

6. Como ejemplo cabe citar que al tratarse de respuestas abiertas, en algunos casos sólo se añade en el apartado de observaciones el país, sin indicar exactamente de qué ciudad, comunidad o región se trata.

7. De la comunidad castellano leonesa es Soria la provincia que aporta un mayor número de estudiantes a esta diplomatura.

8. En el apartado "Otros España” los diplomados indican que se desplazan a trabajar a ciudades como Soria, Valencia o Burgos o expresan sucintamente que lo hacen a la isla de Menorca o a las Canarias, sin concretar la ciudad.

9. Quienes han trabajado en el extranjero indican que lo han hecho en Alemania (Paderborn), en Bélgica y en Irlanda (sin especificar la ciudad en estos dos últimos casos).

10. Además debe tenerse en cuenta que -como se expone en el apartado siguiente- el $7 \%$ de los diplomados está parado y que el $45 \%$ de los que trabajan lo hacen en áreas distintas a las específicas para estos diplomados.

11. En información y documentación se incluyen los trabajos que desarrollan las competencias propias de las tres áreas (biblioteconomía, documentación y archivística).

12. Véase al respecto el último párrafo del apartado anterior.

\section{Bibliografía}

Análisis de la inserción laboral y expectativas profesionales de los graduados en Filosofía y Letras en la Universidad de Zaragoza (1999-2005). Zaragoza: Facultad de Filosofía y Letras, 2005. Consultado en: 31-07-07. http://fyl.unizar.es/doc/Publicacion_egresados_Def.pdf

"Figures on employability of Spanish library and information science graduates" En: Libri, 2001, v. 51, n. 1, pp. 27-37.

Moreiro-González, José-Antonio; Azcárate-Aguilar-Amat, Pilar; Marzal-García-Quismondo, Miguel-Ángel; Tejada-Artigas, Carlos-Miguel; Vergueiro, Waldomiro C. S. "Desarrollo profesional y opinión sobre la formación recibida de los titulados universitarios en información y documentación de las universidades públicas de Madrid (2000-2005)". En: El profesional de la información, 2008, mayo-junio, v. 17, n. 3, pp. 261-272.

Moreiro-González, José-Antonio. "Ajuste de los licenciados en documentación a la oferta de trabajo. Observaciones hechas en la Universidad Carlos III de Madrid”. En: Revista general de información y documentación, 1999, v. 9 , n. 2 , pp. $13-28$

Moreiro-González, José-Antonio; Moscoso-Castro, Purificación; Ortiz-Repiso-Jiménez, Virginia. "El mercado de trabajo de los diplomados españoles en biblioteconomía y documentación”. En: Revista española de documentación científica, 1995, v. 18, n. 4, pp. 444-463.

Tejada-Artigas, Carlos-Miguel; Rodríguez-Yunta, Luis. Situación laboral y desarrollo profesional de los socios de Sedic: la encuesta 2006. Madrid: Sedic, 2006, ISBN 978-611-6225-3.

Tejada-Artigas, Carlos-Miguel; Rodríguez-Yunta, Luis. "Un acercamiento a la situación laboral y desarrollo profesional de los trabajadores de la información y documentación: la encuesta Sedic 2006”. En: X Jornadas españolas de documentación (Fesabid), 2007, pp. 383-391.

Ubieto-Artur, María-Isabel; García-Marco, Francisco-Javier; Domingo-del Valle, María. Evolución de la oferta de empleo público en biblioteconomía y documentación en España durante la década 1996-2006: avance de una investigación. En: X Jornadas españolas de documentación (Fesabid), 2007. pp. 393-401.

\author{
María Domingo-del-Valle, Corporación Aragonesa de \\ Radio y Televisión. \\ mddvalle@unizar.es
}

María-Isabel Ubieto-Artur, Facultad de Filosofía y Letras, Dpto. de Ciencias de la Documentación, Zaragoza.

iubieto@unizar.es

\section{Suscripción EPI sólo online}

Pensando sobre todo en los posibles suscriptores latinoamericanos, ya no es obligatorio pagar la suscripción impresa de EPI para acceder a la online.

EPI se ofrece a instituciones en suscripción "sólo online" a un precio considerablemente más reducido (90 euros/año), puesto que en esta modalidad no hay que cubrir los gastos de imprenta ni de correo postal. 


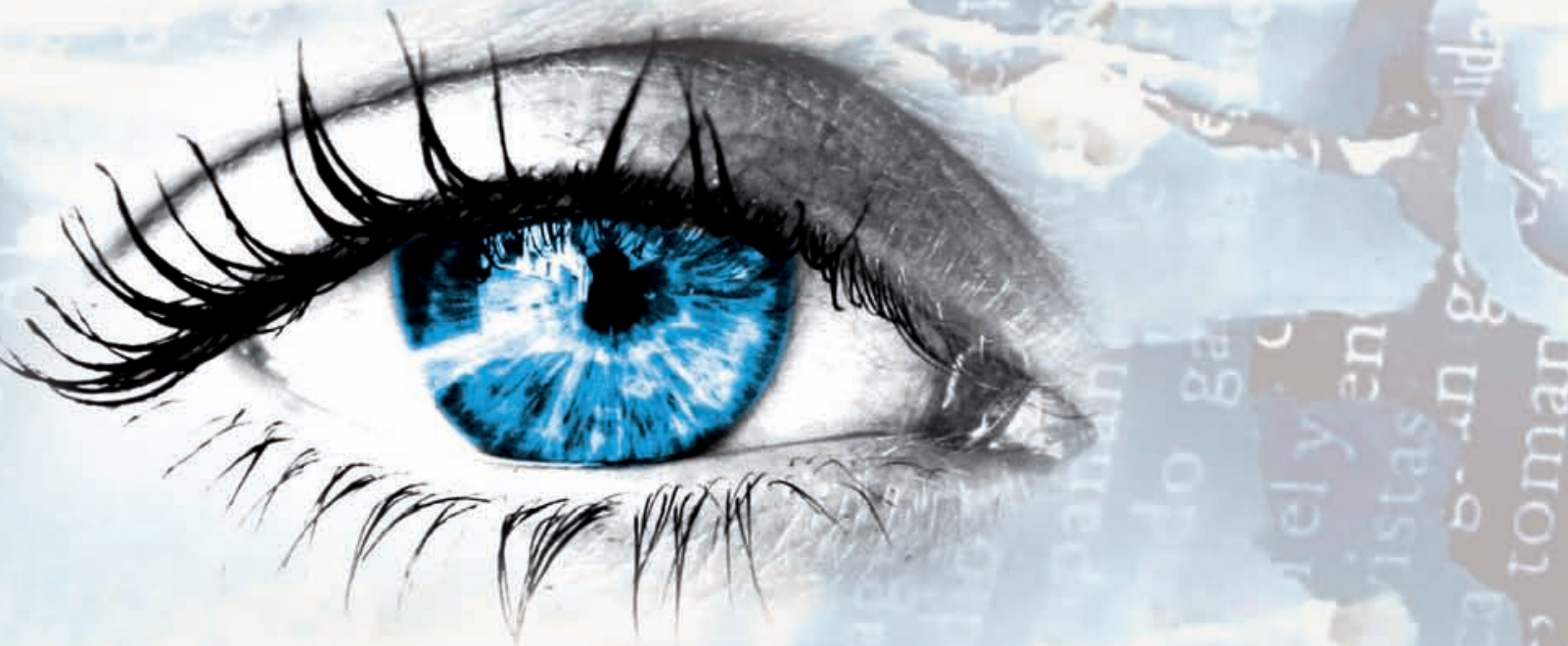

\section{Tenemos otra visión de las bibliotecas.}

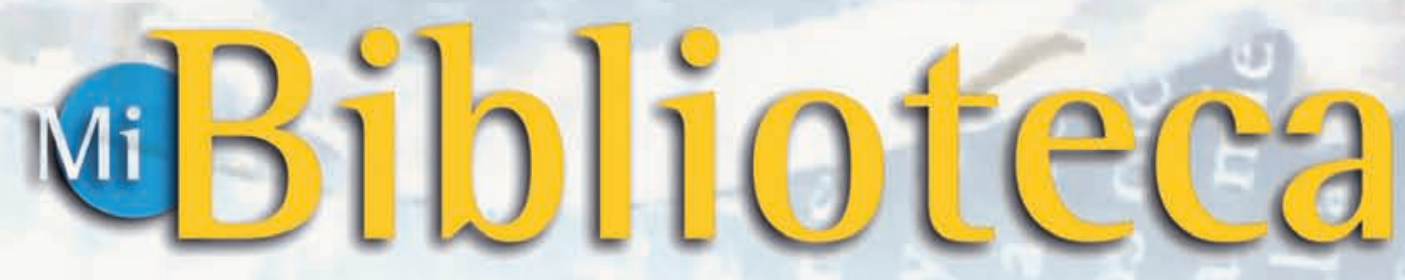

La revista del mundo bibliotecario

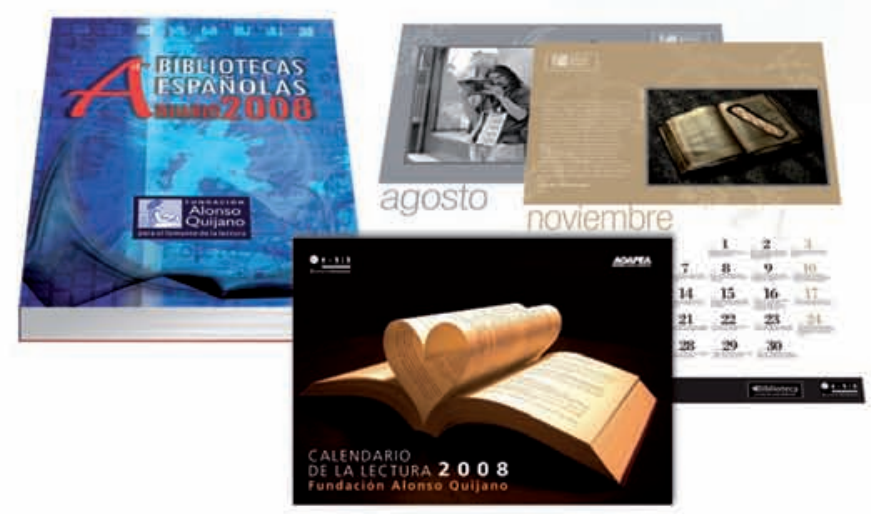

